\title{
KANT, HEREDERO DEL MÉTODO FENOMENOLÓGICO DE LAMBERT*
}

\author{
Claude PICHE \\ Universidad de Montreal
}

RESUMEN: Se trata aqui la influencia que Lambert, y en concreto su Nuevo Organo (1764), ba podido tener en la Dissertatio y en la Crítica de la razón pura de Kant. Este recoge de aquel su preocupación arquitectónica, la recomendación de distinguir entre forma y materia del conocimiento, la de afrontar las contradicciones (el carácter antinómico de la razón) y sobre todo la de distinguir entre la logica de la verdad y la lógica de la apariencia, esencial para comprender el carácter de la metafisica.

Se le da generalmente poca importancia al hecho de que Kant haya pensado primeramente dedicar su Crítica de la razón pura a Johann Heinrich Lambert. Se tiende, en efecto, a olvidar el esbozo de esta dedicatoria, que se encuentra en la número 5024 de las Reflexiones sobre metafísica. Según Adickes, editor de las Reflexiones, este fragmento se remonta a 1776, que corresponde a una fase del itinerario intelectual de Kant en la que trabaja activamente en la elaboración de la primera Crítica. De hecho fue posiblemente la muerte de Lambert, el 25 de septiembre de 1777 , lo que conducirá a Kant a abandonar este proyecto de dedicatoria. Pero ahí queda el hecho de que tuvo la idea, en un momento dado, de colocar el conjunto de su empresa bajo el patronazgo del célebre matemático, astrónomo y filósofo de la Academia de Ciencias de Berlín. La cosa asombra tanto más por cuanto que la Critica de la raźńn pura, por su novedad, incluso por su carácter revolucionario, parece deber bien poco a las obras de los predecesores y de los contemporáneos de Kant. Y el gesto es aún más sorprendente cuando se considera la escasez de las alusiones a Lambert en las obras de Kant, por no hablar ya de su carácter accesorio.

No obstante, si nos volvemos al período que precede al proyecto de dedicatoria, la muy alta estima en la que Kant tenía entonces a Lambert viene a conferir

\footnotetext{
* Traducido del francés por Jacinto Rivera de Rosales, revisado por Enrique Salgado.
} 
una cierta plausibilidad a este proyecto. En la primera carta que le dirige en 1765, ¿no le califica Kant como el "primer genio de Alemania»?' Y cinco años más tarde, en la carta que acompaña el envío a Lambert de un ejemplar de la Dissertatio, ¿no le considera Kant a éste como un interlocutor completamente privilegiado? No sólo apela a su "juicio penetrante» para echar una mirada crítica al opúsculo que le entrega, sino que también le promete hacer llegar, conforme las vaya redactando, las diversas secciones de la metafísica que se propone redactar siguiendo la huella de la Dissertatio. Incluso se compromete ante Lambert a no admitir nada definitivo en esta obra in fieri si eso no le aparece con una "evidencia perfecta" al sabio berlinés ${ }^{2}$. No se podría pedir un testimonio de estima más elocuente.

Sin embargo, de pronto surge una cuestión: si en el curso del perfodo precrítico (que se termina con la carta del 21 de febrero de 1772 a M. Herz) Kant ha tenido el sentimiento de hacer causa común con Lambert, heredero de la tradición racionalista, ¿se puede pensar que, una vez que el proyecto de una Crítica de la razón pura está en construcción, podía Kant todavía pensarse, si no en la letra, sí al menos en el espíritu, laborando en el ámbito del pensamiento de Lambert? ¿no significa el criticismo una ruptura radical y definitiva con la escuela leibniziana? En respuesta a estos interrogantes séame permitido atraer la atención sobre otro hecho, que igualmente no ha sido suficientemente estimado. Se trata del "Anuncio", redactado por Kant, de la publicación del tomo primero del "Deutscher gelehrter Briefwechsel" (Correspondencia de eruditos alemanes) de Lambert. La obra aparece en 1781, es decir, el mismo año que la Crítica de la razbn pura y Kant se toma el trabajo de publicar, con esta ocasión, su "aviso» en un número del Periódico erudito y politico de Königsberg con fecha del 4 de febrero de $1782^{3}$. Es verdad que Kant había estado implicado indirectamente en la correspondencia científica de Lambert, ya que Bernoulli, el responsable de la edición de las obras de Lambert, le había pedido que le hiciera llegar sus propias

${ }^{1}$ Los escritos de Kant están citados por la edición de la Academia de las Ciencias de Berlín (Walter de Gruyter \& Co., Berlín, 1902 ss.), citada como Ak. seguido por el tomo en números romanos y por la paginación en números arábigos. Únicamente la Crítica de la razón pura será citada por la paginación de la primera edición de 1781 (A) y de la segunda de 1787 (B). Para la cita de ahora véase Kant, carta a J. H. Lambert del 31 de diciembre de 1765, en Briefwechsel, Ak. X, 54.

${ }^{2}$ Kant, carta a J. H. Lambert del 2 de septiembre de 1770, Ak. X, 98.

${ }^{3}$ Kant, Anzeige des Lambert'schen Briefwechsel aparecido en la entrega del 4 de febrero de 1782 de los Königsberschen gelehrte und politische Zeitungen, Ak. VIII, 3-4. Para los detalles de la publicación, véase la noticia de Paul Menzer en la edición de la Academia: Anmerkungen der Bände VI-IX, 463-464. 
copias de las cartas intercambiadas con Lambert, a lo que Kant se había negado, pretextando el carácter de inacabado que tenía su contenido. Pero la iniciativa de comentar brevemente en un anuncio el contenido de la correspondencia de este "extraordinario genio" merece en sí mismo ser subrayada ${ }^{4}$. ¿ Se ha de concluir que los escritos de Lambert presentan todavía una actualidad filosófica para el autor de la Crítica de la razón pura?

Confesémoslo ya, los manuscritos de Kant contemporáneos al proyecto de dedicatoria, es decir, los fragmentos póstumos que datan del período que va de 1775 a 1777 , no nos sirven de gran ayuda a este respecto. En efecto, incluso si Lambert es objeto de algunas alusiones en esa época, ellas son demasiado breves como para que uno pueda hacerse una idea precisa de los méritos que Kant está dispuesto a reconocer a este último. Peor aún, Kant se cuida, en cada caso, de atenuar el alcance de los calificativos que utiliza. Así, la Reflexión 4893 conlleva la siguiente mención (aquí en una traducción literal): «Lambert, un analista y arquitectónicon. Detengámonos primeramente en el primer término: sin duda, calificar a Lambert de analista tiene algo de positivo en la medida en que este último se ha opuesto firmemente a Wolf, que colocaba a la base de sus deducciones las definiciones nominales a las que él asentía gratis ${ }^{5}$, antes que investigar pacientemente los elementos primeros del conocimiento a la manera, por ejemplo, de Leibniz. La gran obra de Lambert, el Nuevo Órgano (1764), pretende, por lo demás, retomar con nuevos aportes la characteristica universalis leibniziana ${ }^{6}$. El Kant de la primera mitad de los años sesenta, a saber, el Kant que se interesaba por los conceptos que no pueden ser descompuestos, había descubierto sin duda afinidades con Lambert, el analista ${ }^{7}$. Y más tarde aún, la importancia de un análisis bien llevado podía aparecer como esencial al autor de la Dissertatio de 1770 , en la que se trata de distinguir rigurosamente los elementos del conocimiento sensible de los del conocimiento del mundo inteligible. Pero es evidente que la tarea que se asigna la Crítica de la razón pura va mucho más allá de un simple trabajo de análisis. Eso es al menos lo que per-

\footnotetext{
${ }^{4}$ Kant, carta a Johann Bernoulli del 16 de noviembre de 1781, Ak. X, 278.

${ }^{5}$ Lambert, carta a Kant del 3 de febrero de 1766, Ak. X, 64.

${ }^{6}$ Sobre este asunto véase Claude Debru, Analyse et représentation. De la méthodologie à la théorie de l'espace. Kant et Lambert, Paris, Vrin, 1977, p. 27.

7 Cf. Dieter Henrich, "Kants Denken 1762/3. Über den Ursprung der Unterscheidung analytischer und synthetischer Urteile», en Studien zu Kants philosophischer Entwicklung, $\mathrm{H}$. Heimsoeth et coll. (dir.), Hildesheim, Olms, 1967, p. 17-18.
} 
mite entender la Reflexión 4900: «No me ocupo [...] del análisis como Lambert [...] No estoy en competencia alguna con ese [hombre]".

Lo mismo sucede con el epíteto "arquitectónico» utilizado por Kant para caracterizar el pensamiento de Lambert. Si, en la Dissertatio de 1770 la expresión "genio arquitectónico" encubría aún una connotación peyorativa, este calificativo tomará un sentido mucho más positivo en la segunda mitad de los años setenta, período al que se remonta la Reflexion 4893 (1776-1778) y en el que igualmente Kant se preocupó más de la cuestión del sistema en la filosofía. Un filósofo arquitectónico es, en efecto, aquel que no contempla la parte sino a la luz del todo, a partir de la idea. Es aquel que muestra, en su modo de proceder, originalidad y a la vez amplitud de miras. Lejos de contentarse con juntar las partes del sistema en un agregado, abraza de golpe todo el sistema y se encuentra por ello mismo capacitado para favorecer una actitud autónoma y creativa en sus colaboradores, más bien que restringirlos a tareas serviles ${ }^{8}$. Sin embargo, en la misma época en la que Kant concede a Lambert el título de filósofo "arquitectónico", él formula, en otro contexto, serias reservas en lo que se refiere a la capacidad de este último de dominar la disposición armoniosa de las diversas partes de su gran obra. Estas reticencias están expresadas en una lección sobre la Enciclopedia filosófica de 1775: "Lambert ha [...] escrito un Organon, pero éste se divide y estudia de manera particular cada especie de conocimientos ${ }^{9}$. De hecho Kant deplora aquí la falta de unidad y de cohesión entre las cuatro partes del Nuevo Órgano: 1) la Dianoiologia o doctrina de las leyes del pensamiento, 2) la Aletheiologia o doctrina de la verdad, 3) la Semiologia o doctrina de la designación de los pensamientos y de las cosas, y finalmente 4) la Fenomenología o doctrina de la apariencia. Para completar esta perspectiva sobre los comentarios formulados por Kant acerca de Lambert en el período que va de 1775 a 1777 , se puede citar en último lugar una frase que parece marcar una ruptura profunda entre el pensamiento kantiano en gestación y la figura más tradicional de

${ }^{8}$ Paula Manchester sitúa este período entre 1772 et 1778 , véase su texto "What Kant Means by Architectonicn, en Kant und die Berliner Aufklärung. Akten des IX. Internationalen KantKongresses, tomo 2, V. Gerhardt, R.-P. Horstmann et R. Schumacher (dir.), Berlin, de Gruyter, 2001. 628-630

${ }^{9}$ Kant, Kleinere Vorlesungen und Engünzungen, Ak. XXIX, 32. Véase sobre esto las observaciones aclaradoras de Violetta Waibel, "Natur als 'Aggregat' und 'System'. Kants implizite Auseinandersetzung mit Wolf und Lambert in der 'Ersten Einleitung in die Kritik der Urteilskraft'", en Kant und die Berliner Aufklärung, tomo 4, p. 670. Véase también Giorgio Tonelli, Kant's Critique of Pure Reason within the Tradition of Modern Logic, Hildesheim, Olms, 1994, pp. 48, 87. 
Lambert. Se trata de la Reflexión 4866, que estipula de manera a la vez tajante y lacónica: "Lambert analiza la razón, pero falta aún la crítica»" ${ }^{10}$.

Se ve qué desconcertantes son las afirmaciones de Kant que son más o menos contemporáneas a su esbozo de una dedicatoria de la primera Crítica a Lambert. Aquí, en efecto, se descubre un testimonio excepcional de admiración y de respeto, y allá, un conjunto de observaciones críticas que nos hacen dudar, si no de la sinceridad de Kant en su dedicatoria, al menos de la pertinencia del mensaje implícito en el Anuncio de 1782, según el cual el pensamiento de Lambert habría conservado su actualidad. En lo que sigue, también nosotros nos detendremos a describir la verdadera naturaleza de la influencia ejercida por Lambert sobre Kant, buscando ver en qué medida esta influencia puede ser calificada de durable y definitiva. En verdad, la herencia lambertiana no se sitúa en el plano doctrinal. Es necesario más bien buscar la motivación de la apología kantiana en el gesto filosófico mismo, en la actitud adoptada por Lambert en filosofía. La influencia se sitúa, a decir verdad, más en la manera que en el contenido. Es sabido que Kant reinventa la metafísica, o al menos pone las bases de una metafísica futura. Pues bien, es el método de Lambert lo que Kant juzga como ejemplar en un contexto semejante. Éste será al menos nuestra hipótesis de trabajo en las líneas que siguen. Intentaremos más exactamente mostrar que la herencia lambertiana se sitúa en el ámbito de un método que es preciso calificar de fenomenológico. Para hacer esto, procederemos siguiendo cuatro etapas. (1) La dimensión "metodológica» de la influencia de Lambert sobre la filosofía crítica queda muy bien destacada, como vamos a ver, en el "Anuncio" de 1782 así como en el esbozo de dedicatoria de 1776. (2) En cuanto al carácter "fenomenológico" de este método, él es en verdad el fruto de una lenta gestación en Kant, si bien la «fenomenología general» expuesta en la Dissertatio de 1770 sólo tiene una relación indirecta con el método fenomenológico que se utilizará en la filosofia crítica. (3) Para llegar a ello, Kant debe tener cuenta aún las principales observaciones formuladas por Lambert al hilo de su lectura de la Dissertatio, observaciones que él recibe con la mayor seriedad y a las que presta todo el cuidado requerido, lo cual confirma la altísima estima que tiene por su ilustre colega berlinés. (4) En resumidas cuentas, es desarrollando la Dialéctica transcendental como

${ }^{10}$ Refl. $\$ 4866$, Ak. XVIII, 14. 
una lógica de la apariencia en los años 70 como Kant llega verdaderamente a sacar partido del método fenomenológico de Lambert.

\section{La dimensión metodológica de la influencia de Lambert}

En la carta a Bernoulli del 16 de noviembre de 1781, Kant echa una mirada retrospectiva sobre las relaciones con Lambert y aprovecha la ocasión para poner en claro las afinidades que le ligaban a su colega, del que siente la pérdida. Por supuesto, ambos estaban comprometidos en una «reforma de la metafísica" pero, en el colmo de la ironía, la calidad que de hecho Kant más apreciaba en Lambert era su "inexperiencia" (Unerfahrenheit) en el ámbito de la especulación metafísica. Es lo que confiere una apertura y una gran libertad de pensamiento a ese espíritu esclarecido, inventivo y desprovisto de prejuicios. Esto contrasta, por ejemplo, con un Mendelssohn, que no va en contra de la letra de Baumgarten ${ }^{11}$. Igual que Kant, Lambert posee una vasta cultura científica y es capaz de poner en perspectiva los problemas metafísicos ${ }^{12}$. He ahí lo que habría hecho de Lambert el destinatario privilegiado de la Crítica de la razón pura: a él le habría sido más fácil captar la intención de conjunto, aceptar su novedad y, en su caso, proponer aquí y allá correcciones en vista a perfeccionar y consolidar el sistema ${ }^{13}$.

Por consiguiente, se puede interpretar como un homenaje póstumo el texto del "Anuncio" redactado por Kant con ocasión de la publicación de la correspondencia de Lambert con los sabios. Verdaderamente su apreciación no se dirige al tomo primero de esta correspondencia, sino que este pretexto, por limitado que sea, le ofrece a pesar de todo la oportunidad de señalar aspectos importantes de la obra de Lambert y de hacer resaltar lo que, a sus ojos, es "lo más interesante" en su trabajo ${ }^{14}$. Además del juicio de Lambert sobre el estado de degeneración intelectual del público en materia de gusto, Kant pone de relieve tres rasgos que caracterizan el compromiso intelectual del personaje. Está, en primer

${ }^{11}$ Cf. Marcus Herz, carta a Kant del 11 de septiembre de 1770, Ak. X, p. 100.

12 Además Lambert requiere él mismo de sus lectores esa apertura a diversas ciencias. Cf. Lambert, Neues Organon, Prefacio, p. XIV, en Johann Heinrich Lambert, Philosophische Schriften, tomo 1, H.-W. Arndt (dir.), Hildesheim, Olms, 1965.

${ }^{13}$ Kant, carta a Bernoulli del 16 de noviembre de 1781, Ak. X, 277-278.

${ }^{14}$ Kant, carta a Bernoulli del 22 de febrero de 1782, Ak. X, 280. 
lugar, el diagnóstico esclarecido que hace Lambert sobre las lagunas y los defectos de las ciencias existentes. En segundo lugar, Kant menciona los ensayos y las tentativas de solución propuestas por este último en vistas a remediarlo. Finalmente, en tercer lugar, Lambert se distingue como un sabio que ha buscado indicar pistas para mejorar los métodos de las ciencias y conferirlas todo el rigor deseado. Kant, por supuesto, asume enteramente el primer y el tercer aspecto de la contribución filosófica de Lambert: está claro desde hace mucho tiempo para los dos hombres que la metafísica se encuentra en un estado de crisis, situación que no es posible darle la vuelta sino desencadenando una reforma radical del método. En cuanto al segundo punto, ya hemos afirmado que Kant no retendrá, en definitiva, sino muy poco de las soluciones puntuales aportadas por Lambert a los problemas específicos. Baste aquí mencionar, a título de ejemplo, el juicio muy matizado de Kant sobre los méritos de las innovaciones introducidas por el Nuevo Organo en el capítulo de la lógica ${ }^{15}$. El contenido doctrinal de la obra de Lambert no es manifiestamente la dimensión que él busca poner a la luz. A nuestros ojos, al menos, es más bien el aspecto metodológico de la obra lo que él considera digno de interés.

Este interés de Kant por el método de Lambert se manifestó, por lo demás, muy pronto, es decir, desde el inicio de su intercambio epistolar. Así, ya en su primera carta del 13 de noviembre de 1765 , Lambert se alegra al constatar que ellos dos se encuentran en el mismo camino, en griego se diría métodos ${ }^{16}$. Y Kant le replica manifestando el placer experimentado al constatar la «dichosa coincidencia" de sus respectivos métodos ${ }^{17}$. En sus cartas posteriores, la metodología filosófica, o más precisamente el método particular de la metafísica, se convertirá en un motivo rector constante. Así, Lambert se adhiere a la búsqueda del "método propio de la metafísica" en Kant ${ }^{18}$, reaccionando de la siguiente mane-

${ }^{15}$ He aquí el retrato de Lambert que traza la Lógica editada por Jäsche, Ak. IX, 21: «Del Organo de Lambert se creía ciertamente que iba a ampliar mucho la lógica. Pero no contiene más que divisiones más sutiles que, como todas las correctas sutilezas, agudizan ciertamente el entendimiento, pero son de escaso uso esencial". Cf. los pasajes siguientes de las lecciones de lógica que se refieren a Lambert : Vorlesungen über Logik, Ak. XXIV.1, (Logik Philippi) 338, Ak. XXIV.2, . (Logik Pölitz) 509, (Logik Busolt) 613.

${ }^{16}$ Lambert, carta a Kant del 13 de noviembre de 1765, Ak. X, 51.

${ }^{17}$ Kant, carta a Lambert del 31 de diciembre de 1765, Ak. X, 55.

${ }^{18}$ Kant, carta a Lambert del 31 de diciembre de 1765 , Ak. X, 56. Cf. Kant, carta a Lambert del 2 de septiembre de 1770, Ak. X, 97. 
ra: "Es incontestable que si alguna vez ha de ser encontrada y clarificada una ciencia, esa es la metafísica " ${ }^{19}$. No solamente volverá invariablemente la cuestión del método de la metafísica en su correspondencia posterior, sino que queremos mostrar que las cuestiones de método se encuentran en el corazón mismo de sus intercambios. Tampoco hay que sorprenderse de constatar que Kant está ansioso de enterarse de la reacción de Lambert ante la Dissertatio de 1770. En efecto, después de haber interrumpido durante cuatro años su correspondencia con Lambert, Kant pone fin a su silencia haciéndole llegar su opúsculo, cuya apuesta principal se señala desde el primer parágrafo: «un método de investigación más profunda de las cuestiones metafísicas » ${ }^{20}$.

El esbozo de dedicatoria de 1776 proyectada por Kant para la Crítica de la razón pura es aún más específico en la medida en que indica lo que justamente Kant considera que le debe a Lambert: el «método de la filosofía pura». Sin lugar a duda, Lambert no le ha proporcionado este método en su integridad. Sabemos que Kant, desde ese punto de vista, a roturado pacientemente su propio surco, pero no por ello ha apreciado menos el apoyo que le ha prodigado el que en el fondo se batía por la misma causa: el descubrimiento de una metodología apropiada para la metafísica:

"(para la dedicatoria) Usted me ha honrado con sus cartas. El esfuerzo, a petición suya, de proporcionar un concepto del método de la filosofla pura ha dado ocasión a una serie de observaciones, a que se desarrollaran conceptos que se hallaban en mí aún de manera oscura, y, ampliando y desarrollando las perspectivas, aplazó las respuestas en una dilación interminable. Este escrito [la Crítica de la razón pura] puede servir en el lugar de una respuesta por lo que respecta a la parte especulativa [...] El es atribuible a sus peticiones e indicaciones ${ }^{21}$.

La cuestión que suscita esta dedicatoria es la siguiente: ¿qué balance se puede hacer de los préstamos que Kant ha tomado de su ilustre predecesor? En otras palabras, ¿cómo hay que interpretar estas «indicaciones" (Winke) que el autor del Nuevo Órgano dio a Kant, y de las que hace mención el texto de la dedicatoria? Dejemos por ahora de lado la carta de 1770 , que contiene las observaciones for-

${ }^{19}$ Lambert, carta a Kant del 3 de febrero de 1766, Ak. X, 62.

${ }^{20}$ Kant, De mundi sensibilis atque intelligibilis forma et principits, comúnmente citado como la Dissertatio de 1770, $\$ 1$, Ak. II, 387. Véase ibld., $\$ 23-24$, Ak. II, 410-411.

${ }^{21}$ Kant, Refl. 5024, Ak. XVIII, 64. Cf. Wilhelm S. Peters, «I. Kants Verhältnis zu J. H. Lambert", Kant-Studien, 59, 1968, p. 453. 
muladas por Lambert sobre la Dissertatio, sobre las cuales Kant se excusa en su proyecto de dedicatoria de no haber reaccionado inmediatamente, y retornemos a la carta del 3 de febrero de 1766, en la que Lambert procede a una exposición sistemática de su método. En la enumeración exhaustiva de sus preceptos metodológicos, dos elementos han retenido verosímilmente la atención de Kant: la recomendación, $1^{\circ}$, de distinguir bien forma y materia en el conocimiento, $y$, $2 .^{\circ}$, de no apartarse de las contradicciones que surgen en el medio del camino, siendo lo importante más bien afrontarlas e intentar ver cómo los enunciados contradictorios pueden ser eventualmente reconciliados ${ }^{22}$. Por lo que respecta al primero de esos dos preceptos, hay que confesar que Kant lo ha seguido claramente, como lo señala el título de la Dissertatio: Sobre la forma y los principios del mundo sensible y del mundo inteligible. En cuanto al segundo precepto, él deja entrever ya ese problema característico de la metafísica dogmática que es la antinomia de la razón pura. No es ciertamente por casualidad que Kant, en la carta donde expone a Bernoulli la naturaleza de sus afinidades con Lambert, se toma la molestia de evocar el tema de la antinomia: «las proposiciones [de la metafísica] que con igual derecho pretenden persuadir, se [contradicen] sin embargo en sus consecuencias de una manera tan inevitable que necesariamente se hacen sospechosas las unas a las otras" ${ }^{23}$. Ahora bien, en este mismo pasaje, donde él evoca el conflicto de la razón con ella misma, Kant recuerda a su interlocutor que el conjunto de su empresa tendía en el fondo a descubrir un "criterio cierto de la verdad y de la apariencia». En este contexto hay que confesar que la aparición de la palabra «apariencian no es sin duda inocente.

\section{El concepto de fenomenología en la Dissertatio de 1770}

El problema de la ilusión que acarrea la apariencia ocupa un lugar central en la filosofía de Lambert. El subtítulo del Nuevo Organo no deja, por otra parte, de precisarlo, anunciando con ello la cuarta parte consagrada a la fenomenología:

${ }^{22}$ Lambert, carta a Kant del 3 de febrero de 1766, Ak. X, 63-64.

${ }^{23}$ Kant, carta a Bernoulli del 16 de noviembre de $1781, \mathrm{Ak}$. X, 277. Viene a cuento aquí hacer observar que el esbozo de dedicatoria a Lambert (Reflexion 5024), citada anteriormente, fue escrita por Kant entre las líneas de un fragmento del Prefacio de la Critica de la razón pura donde se trataba, entre otras cosas, la uilusión" engendrada por la antinomia. Cf. Reflexión 5015, Ak. XVIII, 60-61. Véase a este respecto las observaciones de Adickes en la nota al pie de página. 
Gedanken über die Erforschung und Bezeichnung des Wahren und dessen Unterscheidung von Irrthum und Schein [Pensamientos sobre la investigacion y la designación de lo verdadero y su diferencia respecto al error y a la ilusion]. La Fenomenología se asigna también de manera explícita la tarea de delimitar la apariencia y de suministrar los medios para evitar que induzca a error ${ }^{24}$. Incluso siendo la última parte de la obra, Lambert no le concede menor importancia. Al contrario, insiste sobre su carácter de "ciencia fundamental" (Grundwissenschaft). Ciertamente, Lambert no es el inventor del término "fenomenología", como se opina corrientemente, pero él quiere conferir su dignidad filosófica a esta disciplina, de la que se queja por constatar que ha sido desgraciadamente demasiado poco practicada ${ }^{25}$.

«De la fenomenología poco ha aparecido aún hasta ahora en las doctrinas de la razón, a pesar de ser tan necesario distinguir lo verdadero de la ilusión. Ella no se dirige ciertamente de manera directo a la llamada verdad lógica, sino más a la verdad metafisica, porque la ilusión se contrapone mayormente a lo reabs ${ }^{26}$.

A partir de 1764, Kant tenía pues ante sus ojos el modelo de una disciplina explícitamente consagrada a la solución de los problemas metafísicos. No es porque Lambert haya forjado esta ciencia sobre el modelo de la óptica científica, la cual explica el origen de las ilusiones sensibles, que la apariencia en cuestión se limita a los engaños de los sentidos. Lambert habla más bien de una óptica "transcendente", subrayando con ello que la óptica debe también ser comprendida en un sentido metafórico ${ }^{27}$ : la fenomenología juega un papel análogo a la ciencia de la óptica cuando aparta los prestigios y los errores de la metafísica. Con toda verosimilitud se puede interpretar como una reminiscencia la comparación que hace Kant al principio de la Dialéctica de la Crítica de la razón pura entre la apariencia transcendental y las ilusiones de la óptica. Por ahora, no obstante, conviene examinar la manera como Kant ha integrado progresivamente el paradigma fenomenológico en su filosofía.

${ }^{24}$ J. H. LAMBerT, Neues Organon, Prefacio, p. IV.

${ }^{25}$ J. H. LAMBERT, Neues Organon, dans Philosophische Schriften, Tome 2, p. 218 ; trad. fr. de la sección IV del Nouvel Organon: "Phénoménologie ou doctrine de l'apparence». por G. Fanfalone, Paris, Vrin, 2002, p. 32. Véase la nota del traductor en su Introducción (p. 14), según la cual es el teósofo pietista Christoph Friedrich Oetinger al que hay que atribuir la primera aparición (1736) del término "pha[i]nomenologia".

${ }^{26}$ J. H. LAMBERt, Neues Organon, Prefacio, p. XI-XII, subrayado por Lambert.

${ }_{27}$ Véase sobre este asunto Lewis WHITE BECK, Early German Philosophy. Kant and his Predecessors (1969), Bristol, Thoemmes Press, 1996, p. 408. 
Se puede comprender el entusiasmo de Kant cuando le hace llegar a Lambert su Dissertatio. Él cree haberse inspirado tan bien en el método de éste último, que es capaz de pretender que su texto es "fenomenológico" de un extremo al otro. Así, en la carta del 2 de septiembre de 1770, que acompaña a su envío, él declara que, en lo esencial, su trabajo consiste en una phaenomenologia generalis. Sin embargo, se podría creer que recurre a esa expresión simplemente para complacer a su destinatario. Pero el hecho es que la retomará dos años más tarde en su célebre carta a Marcus Herz del 21 de febrero y, de nuevo, para designar lo esencial del contenido de la Dissertatio ${ }^{28}$. Kant la concibe como una "propedéutica", teniendo por objeto "preservar" la metafísica, como le dice a Lambert, de toda contaminación con la forma y los principios del mundo sensible, eliminando a la vez las múltiples posibilidades de equivocación. La fenomenología general contenida en la Dissertatio tiene, en el fondo, únicamente una utilidad negativa: ella cerca con precisión el campo del conocimiento fenoménico para oponerse a toda tentativa de aplicar los principios del mundo sensible al mundo inteligible.

"Las leyes más generales de la sensibilidad juegan falsamente un gran papel en la metafisica, donde todo depende sin embargo de los conceptos y principios de la razón pura. Parece que una ciencia enteramente especial, aunque simplemente negativa (phaenomenologia generalis) tiene que preceder a la.metafísica. En esa ciencia se le determinarán a los principios de la sensibilidad su validez y sus límites, a fin de que no perturben los juicios sobre los objetos de la razón pura, como ha sucedido casi siempre hasta ahora ${ }^{29}$.

La fenomenología de la que aquí se trata no es, como en Lambert, una ciencia que verse directamente sobre la apariencia engañosa presente, en esta ocasión en la metafísica, sino más bien una ciencia que precede a la metafísica en el sentido en el que ella no versa sino sobre el conocimiento del mundo sensible, es decir, sobre su forma y sus principios legítimos. Veremos más adelante que Kant sacará provecho del método fenomenológico de Lambert para la denuncia propiamente dicha de la ilusión metafísica. Pero en 1770, Kant no ha llegado aún a una concepción clara de las fuentes de la apariencia dialéctica.

${ }^{28}$ Kant, carta a Marcus Herz del 21 de febrero de 1772, Ak. X, 129. Cf. Reflexión. 4163, de 1769-70, Ak. XVII, 440.

${ }^{29}$ Kant, carta a Lambert del 2 de septiembre de 1770, Ak. X, 98. 
Sin duda, el tema de la apariencia engañosa está presente en el texto de la Dissertatio en relación, por ejemplo, con los principios usurpados (sub specie axiomatum $)^{30}$, pero no está enlazado en cuanto tal a la fenomenología. Es decir, que Kant, y eso sorprende en un primer momento, presume ante Lambert de retomar por su parte el concepto de fenomenología, pero para hacer de él un uso diferente a la descripción que se encuentra en el Nuevo Órgano, o sea, sin referencia directa a una apariencia susceptible de inducir a error. En efecto, si la palabra "apariencia" interviene en la Dissertatio es bajo el término latino apparentia, aplicado indistintamente al conjunto del mundo sensible. Es lo que se convertirá en la primera Crítica en Erscheinung [fenómeno]. Esto significa que mientras los principios de lo sensible no sobrepasen los límites del mundo fenoménico, los conocimientos que permiten alcanzar deben ser considerados como "muy verdaderos" ". Este uso positivo del término "fenomenología", sin referencia alguna a una ilusión especiosa, permanecerá en Kant, incluso a lo largo del decenio de las tres Críticas, en el que, por ejemplo, los Primeros principios metafisicos de la ciencia de la naturaleza darán un nuevo sentido a esa palabra, pero exento también de toda referencia a la ilusión dialéctica ${ }^{32}$.

Se puede fácilmente comprender la reacción negativa de Lambert a esta generalización de la apparentia al nivel del conocimiento sensible, reacción bien legítima y sobre la que tendremos ocasión de volver. Por mucho que se esfuerce Kant en precisar que la apparentia constituye el elemento de base de toda nuestra experiencia, ¿̨no amenaza el término con hacer de la totalidad del mundo sensible una ilusión? Por ahora lo que importa es, no obstante, precisar el alcance crítico que

${ }^{30}$ KANT, Dissertatio de 1770, $\$ 23$, Ak. II, 411.

${ }^{31}$ KaNT, Dissertatio de 1770, $\$ 11,15$, Ak. II, 397, 404. La traducción de Norman Kemp Smith del término Erscheinung al inglés por appearance no es tan incongruente como parece a primera vista, al menos en cuanto que se vea en la apariencia una alusión a la apparentia de la Dissertatio más que una traducción del Schein. Cf. A Commentary to Kant's 'Critique of Pure Reason', New-York, Humanities Press, 1950, p. 83.

${ }^{32}$ Como lo ha hecho notar acertadamente Claude Debru en Analyse et représentation, p. 152, la definición siguiente de la fenomenología comporta un distanciamiento inequívoco frente a la concepción lambertiana: "Aquí no se trata de una transformación de la ilusión en verdad, sino del fenómeno en experiencia; pues en el caso de la ilusión el entendimiento siempre juega un papel, con sus juicios que determinan un objeto, si bien corre el peligro de tomar lo subjetivo por objetivo; pero en el fenómeno no se encuentra ningún juicio del entendimiento; lo cual ha de ser tenido en cuenta no sólo aquí, sino en toda la filosofía, porque, en caso contrario, si se habla de fenómeno y se toma esa expresión con el mismo significado que ilusión, siempre será mal comprendido" (Kant, Metaphysische Anfangsgründe der Naturwissenschaft, Ak. IV, 555) 
Kant confiere a la fenomenalización del mundo de la experiencia ${ }^{33}$. Haciendo del fenómeno el objeto de la intuición sensible, cuya forma es espacio-temporal, Kant pretende salvaguardar un lugar teórico que escape a esta intuición y donde pudieran encontrar su lugar los objetos de la metafísica, incluso si el ser humano no puede acceder a otro tipo de intuición que la sensible. Kant anticipa con ello el motivo subyacente al capítulo de la Critica de la razón pura consagrado a la distinción entre fenómeno y noúmeno, en el cual toda la apuesta consiste en limitar las pretensiones de la sensibilidad y en reservar un ámbito para los objetos (noumena) susceptibles de ser aprehendidos en su caso por una intuición intelectual $^{34}$. Como se ve, igual que en la Crítica, aquí nos las habemos con un intento de delimitación que no tiene aún nada que ver con la temática propia de la Dialéctica transcendental: la producción de la apariencia por la razón misma.

Lo mismo se podría decir de otro tema muy presente en la Dissertatio, a saber, la tarea de poner al mundo inteligible al abrigo de la contaminación de los principios del mundo fenoménico. Una vez más, el tema del phaenomenon intellectuatum no deja de recordar a otro capítulo de la primera Crítica que, al igual que el capítulo consagrado a la distinción entre fenómeno y noúmeno, precede a la Dialéctica y, por esa razón, está mas ligado a las prerrogativas del entendimiento que a la de la razón propiamente dicha. Naturalmente, el desdoblamiento del intellectus en entendimiento y razón no existe aún en el tiempo de la Dissertatio, pero este hecho sirve precisamente de indicio de la inexistencia, en ese texto, de una problemática específica de la razón: una ilusión dialéctica, cuyo origen hay que buscarlo en los principios mismos de esta facultad de conocer vuelta, gracias a la idea, hacia lo incondicionado, hacia lo absoluto ${ }^{35}$. Como esta

${ }^{33}$ KANT, Dissertatio de $1770, \wp 5$, Ak. II, 394.

34 «Suponemos, en efecto, con razón que todo lo que no puede ser conocido claramente por ninguna intuición no es pensable, y por tanto es imposible. Pero de que no podamos, por ningún esfuerzo del espíritu, ni siquiera recurriendo a la ficción, llegar a otra intuición que a la que se hace según la forma del espacio y del tiempo, de ello concluimos en general que toda intuición que no está sujeta a esas leyes es absolutamente imposible (olvidando la pura intuición intelectual, exenta de las leyes de los sentidos, como es la intuición divina, que Platón llama idea), y entonces sometemos a todas las cosas posibles a los axiomas sensibles del espacio y del tiempo" (Kant, Dissertatio de $1770, \$ 25$, Ak. II, 413).

${ }^{35}$ Para el surgimiento progresivo de la Dialéctica transcendental, y en particular de la antitética de la razón pura, en los anios 70, se puede consultar las Reflexiones siguientes en Ak. XVII y XVIII: 4454 (1772-75 ?), 4469 (1772), 4762 (1775-76), 4952 (1776-78), 4985 (1776-78) y 5058 (177678). El desarrollo de la teoría de la ilusión transcendental va a la par evidentemente de la distinción 
problemática está aún latente en la Dissertatio, Kant puede tratar ahí los problemas de la dialéctica sólo desde el punto de vista de la interferencia de los principios del mundo sensible en el conocimiento intelectual. Así, del mismo modo que en el capítulo de la Crítica de la razón pura titulado "Anfibología de los conceptos de la reflexión», Kant reprocha a Leibniz el haber «intelectualizado» los fenómenos, y a Locke el haber "sensualizado" los intellectualia ${ }^{36}$, él aborda aquí el tema de los "fenómenos intelectualizados» describiendo su ilegitimidad de la manera siguiente:

"Pero pudiéndose llamar vicio de subrepción las ficciones del intelecto producidas por la intromisión de un concepto sensible como si fuera una nota intelectual, así la permutación de lo intelectual por lo sensible será vicio metafísico de subrepción (fenómeno intelectualizado, si es permitida la bárbara expresión), y por lo mismo tal axioma hibrido, que proclama lo sensible como necesariamente adscrito a lo inteligible, lo llamo axioma subrepticio ${ }^{37}$.

Sin duda, en esta andadura Kant está claramente preocupado por la suerte de la metafísica y por la génesis de la ilusión que viene a comprometer su estatuto de ciencia, pero se estará de acuerdo en que la explicación del nacimiento de la apariencia está mucho menos desarrollado que en la Crítica de la razón pura, lo que hace que Lambert tenga dificultad en encontrar en la Dissertatio, más allá del uso tan particular que Kant haçe del término de fenomenología -él lo reserva a un dominio del conocimiento "muy verdadero", el del mundo sensible- el espíritu de esta disciplina: distinguir en la apariencia lo que hay de real y de ilusorio.

Es, pues, debido a que los cuatro temas ligados a la idea cosmológica (infinitud espacio-temporal, simplicidad, milagro y causa del mundo) están presentes en la Dissertatio por lo que es preciso concluir que Kant está en plena posesión de lo que llegará a ser la antitética de la razón pura ${ }^{38}$, figura gracias a la cual la ilusión provo-

neta entre el fenómeno (Erscheinung) y la ilusión (Schein). Cf. también Kant, Vorlesungen über Metaphysik, Ak. XXVIII, (Metaphysik L1) 234. Véanse además las Reflexionen siguientes sobre la lógica en Ak. XVI, Reflexiones 2249, 2250, 2254, y sobre la metafisica en Ak. XVIII, 4930 (1776-78) y 4999 (1776-78). Se puede consultar finalmente Eric Watkins, "The 'Critical Turn': Kant and Herz from 1770 to 1772», en Kant und die Berliner Aufklärung, tomo 2, p. 77.

${ }^{36}$ Kant, Critica de la razón pura, A 271/B 327.

${ }^{37}$ KANT, Dissertatio $\$ 24$, Ak. II, 412.

${ }^{38}$ Kant, Dissertatio, $\$ 28,30$, Ak. II, 416, 418. La concepción de las ilusiones del entendimiento (praestigiae intellectus) expuesta en la Dissertatio difiere algo de lo que será la concepción de la ilusión dialéctica en la primera Crítica. Asi, por ejemplo, en 1770 hay ciertamente un conflicto 
cada por el conocimiento metafísico se manifiesta de la manera más espectacular. Para llegar a ello, Kant deberá atravesar numerosas etapas, comenzando por el descubrimiento del origen de la ilusión en otra parte que en la interferencia pura y simple de los principios del conocimiento sensible en el conocimiento inteligible. No basta limitar el conocimiento sensible en sus pretensiones, es necesario además ver que el conocimiento del mundo inteligible tiene, en él mismo, necesidad de limitaciones. Este rasgo aparece por lo demás en los títulos proyectados por Kant para su gran obra: así, en su carta a Herz de 1771, se hará cuestión de ahí en delante de los límites del conocimiento, comprendidos los del conocimiento inteligible: Los límites de la sensibilidad y de la razbn, título que en 1772 se transformará para poner toda la atención sobre el conocimiento racional: Critica de la razón pura ${ }^{39}$. Pero para llegar a la obra publicada en 1781, será necesario que Kant deje madurar en él el verdadero sentido del método fenomenológica según Lambert. Y este último, en la carta que contiene sus reacciones ante la Dissertatio, no dejará de recordárselo.

\section{La respuesta de Lambert}

Entre las numerosas observaciones formuladas en esta carta del 13 de octubre de 1770 , podemos considerar un reproche y un consejo. El reproche atañe al peligro de caer con el fenomenalismo de la Dissertatio en un idealismo radical, mientras que el consejo consiste en recordar el método que exige esta disciplina tan particular que es la metafísica: el método fenomenológico, de lo que, por lo demás, los astrónomos ofrecen el ejemplo en su propio modo de proceder.

La advertencia contra el idealismo cubre una buena parte de las observaciones de Lambert, y en él se apoya en una profunda convicción que no ha dudado en exponer en la Fenomenologid ${ }^{40}$ : para él, en efecto, no se trata de reducir la temporalidad y la extensión a la pura fenomenalidad, es decir, según él, a una simple ilu-

e incompatibilidad (reluctantia, repugnantia) entre los principios del mundo sensible y los del mundo inteligible. Pero el hecho de que el conflicto encuentra su solución cuando es situado en el sujeto más bien que en la cosa misma, no es sino una tímida anticipación de la teoría de la antinomia de la razón pura. Cf. Dissertatio $\$ 1,24,26$, Ak. II, 389, 412, 413.

${ }^{39}$ KANT, carta a Marcus Herz del 7 de junio de 1771 , Ak. X, 123. Cf. carta a Marcus Herz del 21 de febrero de 1772, Ak. X, 129, 132.

${ }^{40}$ LAMBert, Neues Organon, Phänomenologie, $\$ 9$, 21, en Philosophische Schriften, t. 2, p. 222-223, 230. Vease igualmente la carta de Lambert a Holland citado en C. Debru, Analyse et représentation, p. 55 . 
sión (blosser Schein). Al contrario, el tiempo y el espacio tienen, a sus ojos, una realidad indudable. De ahí, sin duda, su estupefacción ante la tesis de la Dissertatio según la cual estas dos formas de la intuición son ellas mismas, dos "fenómenos", o sea, seres de la imaginación (entia imaginarium) ${ }^{41}$. Él no podía, por tanto, comprender de otra manera la expresión utilizada por Kant para designar la Dissertatio, phaenomenologia generalis, que reduce la integridad de la experiencia sensible a una apariencia. El argumento concerniente a la irrealidad de los cambios en el tiempo, tal y como está presentado en la carta de Lambert, sólo expresa una de las consecuencias de este idealismo: si el tiempo es una simple forma ideal de la sensibilidad propia del sujeto, esto implica que los cambios, que se desarrollan en el tiempo, participan de la misma irrealidad de aquél. No tienen por tanto nada de objetivo. Kant confiesa, por su parte, que ha tomado seriamente este reproche. Más tarde confesará a Bernoulli que el pasaje A 36-38 de la Crítica de la razón pura se dirige en el fondo a responder a la objeción de Lambert ${ }^{42}$.

Por detrás de ese reproche, concerniente al estatuto del cambio en el tiempo, se perfila con toda evidencia el rechazo por parte de Lambert de esta fenomenología general kantiana, que constituye un formidable malentendido respecto a la intención primera del Nuevo Órgano. Por medio de la fenomenología, Lambert no ha intentado jamás transformar el mundo de la experiencia en una pura ficción, por muy coherente que ésta fuera. Al contrario, en bastantes casos la apariencia puede ser fundada y en ese caso se convierte en una apariencia "real", perdiendo por ello todo el carácter ilusorio, o sea, su mismo estatuto de apariencia.

"Tiempo y espacio serán apariencia real, a cuya base yace algo que se rige tan exacta y constantemente según la apariencia como exactas y constantes pueden ser siempre las verdades geométricas [...] Pero he de decir que una tal apariencia que nunca engaña habrá de ser algo más que simplemente apariencia» ${ }^{43}$.

41 KaNT, Dissertatio $\$ 14,15$, Ak. II, 401,404.

42 KANT, carta a Marcus Herz del 21 de febrero de 1772, Ak. X, 134. Carta a Bernoulli del 16 de noviembre de 1781, Ak. X, 277. De todos modos, conviene señalar que la tesis de la Dissertatio, acerca de la idealidad del espacio y el tiempo, provocó un coro de protestas, especialmente las de Sulzer, Mendelssohn y Schutz, de modo que el pasaje de la Critica se dirige, de manera general, a responder a estos einsehende Männer [hombres perspicaces]. Cf. Carta de Sulzer a Kant del 8 de diciembre de $1770 \mathrm{Ak}$. X, 112. Carta de Mendelssohn a Kant del 25 de diciembre de $1770 \mathrm{Ak}$. X, 115. Sobre el predicador de la Corte Schultz, véase Carta de Kant a Marcus Herz del 21 de febrero de 1772, Ak. X, 133. Kant Critica de la Razón Pura A36/B53. Sobre este asunto véase el artículo de Arthur Melnick, «Kant vs Lambert y Trendelenburg on the Ideality of Timex History of Philosophy Quarterly, 18, 2001 pp. 79-90.

${ }^{43}$ Lambert, carta a Kant del 13 de octubre de 1770, Ak. X, 110. 
De hecho, Kant podría considerarse completamente protegido ante el reproche de idealismo esbozado aquí, pues el mundo fenoménico, tal y como él lo entiende, es igualmente para él muy real. Anticipando la acusación de idealismo, el $\$ 11$ de la Dissertatio había especificado efectivamente que los fenómenos no son simples quimeras en la medida en que, desde el punto de vista metafísico, poseen un auténtico fundamento. Ya que ellos "dan testimonio de la presencia de un objeto", no tienen nada que ver con entidades fantasmales. Como lo afirma Kant, los fenómenos del mundo sensible están fundados (causata) ${ }^{44}$. En esas circunstancias, es diff́cil concebir que se haya podido sentir desconcertado por los argumentos de Lambert.

En lo que respecta, por otra parte, al consejo prodigado a Kant en la carta que aquí se está analizando, debemos precisar primeramente que él se inscribe en el marco de un segundo reproche, que el célebre comentarista de Kant, Lewis White Beck, considera como el elemento de más hondas consecuencias en esta respuesta de Lambert. Se trata de la recomendación hecha a Kant de superar la estricta «separación" ocasionada por la «heterogeneidad" de las fuentes del conocimiento sensible y del conocimiento inteligible, y de no dudar en aplicar los conceptos ontológicos (del entendimiento puro) a los fenómenos. No discutiremos aquí esta tesis de Beck ${ }^{45}$, pero prestaremos sin embargo atención a un aspecto subrayado de pasada por este comentarista: el recuerdo del método del astrónomo. Se trata, en verdad, de uno de los temas favoritos de Lambert, tema del que se sirve abundantemente en su Fenomenologia para ilustrar sus propio procedimiento ${ }^{46}$ : el astrónomo toma su punto de partida en los "fenómenos" tal como ellos se presentan —así, por ejemplo, cuando un astro adopta una trayectoria imprevista - él va a la búsqueda de las leyes generales inspiradas por sus

${ }^{44}$ KanT, Dissertatio $\$ 11$, Ak. II, 397.

45 Lambert, carta a Kant del 13 de octubre de 1770, Ak. X, 105. Lewis White Beck, "Lambert und Hume in Kants Entwicklung von 1769-1772", Kant-Studien, 66, 1969, 123-130. Para Beck, la exhortación hecha a Kant de aplicar los conceptos ontologicos a la esfera de los fenomenos prefigura la pregunta central de la famosa carta a Herz del 21 de febrero de 1772: ¡cómo es posible una representación que se refiera a un objeto sin ser de alguna manera afectado por él? Beck es de la opinión de que es Lambert quien ha atraído la atención de Kant sobre este problema, cuya solución estará en el centro del trabajo de Kant durante el resto de los ańos setenta. Es preciso darse cuenta, sin embargo, que en el manuscrito de Duisbourg, por ejemplo, no se hace referencia alguna a Lambert.

${ }^{46}$ Lambert, Neues Organon, Phänomenologie, $\$ \$ 2,4$, 163, 167, Philosophische Schriften, Tome 2, 218, 220, 330, 337. Véase ibíd., Dianoiologie, \579, Philosophische Schriften, tomo 1, 368. 
observaciones y las aplica a su vez a los fenómenos. Pues bien, en su carta de 1770, Lambert se preocupa de recordarle a Kant que este método, que no dudamos en calificar de fenomenológico, está particularmente indicado para la metafísica, disciplina que se ve confrontada muy a menudo con la apariencia.

«En la metafísica, donde la dificultad de la apariencia tiene tanta presencia, el método de los astrónomos es ciertamente el más seguro. El metafísico puede tomar todo como apariencia, separar las vanas de las reales, y concluir lo verdadero a partir de la apariencia real ${ }^{47}$.

Es legítimo preguntarse cómo Kant ha podido reaccionar a esta recomendación. En virtud de su propio concepto de fenomenología generalizada, Kant no se plantea transformar el mundo sensible en una vasta ficción. Pero he aquí que Lambert le recuerda su concepción de la fenomenología, hecha a media, por lo demás, como se acaba de ver, para las cuestiones metafísicas, donde precisamente la apariencia plantea tantos problemas. En otras palabras, ¿podía Kant reconciliar su concepto de fenomenología, es decir, el fenomenalismo generalizado a todo el mundo sensible, con el método fenomenológico de Lambert y producir, a partir de ahí, un sistema filosófico coherente?

\section{El fenómeno en un sentido nuevo: la antinomia}

La respuesta directa a esta cuestión no se ha de buscar en la Crítica de la razón pura, sino en los Prolegómenos, donde se descubre un pasaje sumamente elocuente sobre la conciencia que puede tener Kant de la distancia que separa su propio concepto de fenomenología de aquél, claramente metodológico, de Lambert, así como de la necesidad de echar mano de los dos en el seno de la filosofía crítica. En el pasaje que sigue, es necesario prestar atención en sentido que recibe el término Schein [apariencia, ilusión], que no debe ser identificado con el carácter fenoménico del mundo expuesto en la Dissertatio, sino más bien con la apariencia propiamente metafísica, de la que la Crítica saca a la luz el mecanismo de su surgimiento. $Y$ sin embargo, carácter fenoménico y apariencia o ilusión transcendental son elementos ligados en el criticismo.

"En segundo lugar, estos principios míos, lejos de convertir las representaciones de los sentidos, al hacer de ellas fenómenos, en mera apariencia ilusoria en

${ }^{47}$ Lambert, carta a Kant del 13 de octubre de 1770, Ak. X, 108. 
lugar de la verdad de la experiencia, son más bien el único medio de impedir la ilusión transcendental, con la cual se ha engañado siempre la metafísica y ha sido conducida a esfuerzos pueriles por atrapar pompas de jabón, precisamente porque se tomaban los fenómenos, que son sin embargo meras representaciones, por cosas en sí mismas; de lo cual resultaron todas aquellas curiosas manifestaciones de la antinomia de la razón, a la que me referiré luego, y que queda suprimida con aquella única observación: que el fenómeno, mientras se lo usa en la experiencia, produce verdad, pero en cuanto traspasa los límites de la experiencia y se vuelve transcendente, no produce sino mera apariencia ilusorias ${ }^{48}$.

No cabría pedir una cita más explícita para ilustrar la necesidad de mantener uno al lado del otro los dos conceptos de fenomenología: no es sino gracias al trabajo preparatorio de la Dissertatio que Kant se ha puesto en condición de tematizar y de resolver el enigma de esta ilusión transcendental que se manifiesta de manera paradigmática en la antinomia. La fenomenología generalizada de Kant se ha mostrado, en efecto, indispensable, a pesar de la sospecha de idealismo que pesa constantemente sobre ella, para desactivar la ilusión dialéctica. El extracto citado antes es, pues, un texto de excepcional interés, en el que es preciso ver una respuesta definitiva a las inquietudes y demandas de elucidación de Lambert en su carta, o incluso una reconciliación final entre los dos filósofos.

Pero entonces se estaría autorizado a afirmar que Kant, en este pasaje, como tampoco en los otros textos de su filosofía de la madurez, no confiesa su deuda respecto a Lambert, como tampoco le da el nombre de "fenomenología" al método que él despliega en la Dialéctica transcendental. Estas dos reservas están en parte fundadas porque, en un primer momento, es verdad que no se puede descubrir ninguna influencia directa de Lambert en el método puesto en práctica por Kant en su enfoque de la metafísica dogmática. ¿ Se parece este enfoque efectivamente al del astrónomo, que toma su punto de partida en la apariencia, incluso engańosa, a fin de producir una explicación? Para esto sería necesario que Kant utilice la palabra "fenómeno" no en la acepción que él mismo le da en su Estética transcendental, sino en la de Lambert. Pues bien, tal uso existe ciertamente en la Crítica de la razón pura, como vamos a verlo más adelante. Pero por ahora con-

${ }^{48}$ Kant, Prolegomenos $\$ 13$, Observación. III, Ak. IV, 292. Desde luego, en los Prolegómenos Kant toma nota de la acusación de idealismo contenida en la recensión de Garve-Feder y procura hacerle frente sin rodeos. Pero la necesidad de distinguir nítidamente el fenómeno (Erscheinung) de la apariencia (Schein) siempre le resultó clara. Véase igualmente Crítica de la Razón Pura B69. 
viene acudir de nuevo al texto de los Prolegómenos, donde Kant se expresa con una claridad inaudita sobre el modo de proceder de la Dialéctica transcendental, gracias a la distancia que es capaz de tomar, en 1783, respecto a la Critica. El pasaje que sigue, y no es el único, hace mención del concepto de fenómeno en un sentido nuevo: él designa lo que moviliza la atención, lo que atrae la mirada sobre él, sin tener que ser por eso un fenómeno relevante de la forma y de los principios del mundo sensible (Erscheinung). Eso es tan verdadero que en este caso se trata de un fenómeno de la razón humana (Phänomen der menschlichen Vernunft), más precisamente de un fenómeno excepcional, que ha contribuido en suma a despertar a Kant de su sueño dogmático: la antinomia. Y para designar esta manifestación, Kant usa la palabra "Phänomen». en un sentido especial. Si en el momento de la Dissertatio, la antitética de la razón pura no había sido todavía elaborada en toda su amplitud, he aquí que Kant, en 1783, es capaz de decir, de manera casi explícita, que ella es el fruto de la aplicación de este método fenomenológico preconizado por Lambert:

"He aquí, pues, el fenómeno (Phänomen) más extraño de la razón humana, del cual no se puede mostrar ningún otro ejemplo en ningún otro uso de ella. Cuando pensamos, como ocurre habitualmente, los fenómenos del mundo sensible como cosas en sí mismas, cuando tomamos los principios de su enlace como principios válidos universalmente para cosas en sí mismas y no solamente para la experiencia [...] entonces se manifiesta un conflicto insospechado, que nunca puede ser superado por el camino dogmático habitual, porque tanto una proposición como su contraria pueden ser demostradas mediante pruebas igualmente evidentes, claras e irresistibles [...] y la razón se ve así dividida consigo misma; una situación sobre la que el escéptico se regocija, pero al filósofo crítico le ha de inducir a la reflexión y provocarle inquietud " $"$.

Como se ve, es este fenómeno de la antinomia, el fenómeno «más notable» de los que hay ${ }^{50}$, el que le ha puesto a Kant en el apuro y le ha suscitado sus reflexiones. Es verdad que el procedimiento crítico es único y es posible pretender que sólo tiene un parentesco lejano con el método fenomenológico que Lambert toma prestado de los astrónomos. ¿Son suficientes dos casos de esta acepción de la palabra fenómeno en los Prolegómenos para acreditar esta influencia?

\footnotetext{
49 KANT, Prolegomenos $\$ 52$ a, Ak. IV, 339-340.

${ }^{50} \mathrm{KANT}$, Prolegomenos $\$ 50$, Ak. IV, 338.
} 
Pues bien, si se consideran las cosas más de cerca, se comprueba que la Crítica contiene también dos casos de la palabra "fenómeno" en ese sentido inusitado, y precisamente en el capítulo sobre la Antinomia. El primero de esos dos es el siguiente:

"Aquí, en efecto, se muestra un nuevo fenómeno (Phänomen) de la razón humana, a saber, una antitética enteramente natural, para llegar a la cual nadie necesita hacer sutilezas ni poner trampas artificiales, sino que la razón cae en ella por sí misma y en concreto de manera inevitable. Gracias a ella, la razón se mantiene al resguardo del sopor de una convicción ficticia, producida por una apariencia ilusoria meramente unilateral $" 51$.

Este pasaje tiene de interesante el que precisa la naturaleza específica del fenómeno-apariencia en la idea cosmológica: no se trata, como es el caso de las otras dos ideas, de una apariencia "unilateral" (einseitig). Así, por ejemplo, en su capítulo sobre el ideal transcendental, Kant calificará nuevamente la apariencia que obra en las pruebas de la existencia de Dios como Phänomen des Verstandes [fenómeno del entendimiento] ${ }^{52}$. Esto es consecuente con el método fenomenológico desplegado en el conjunto de la Dialéctica. Pero la particularidad de la Antinomia, de donde procede su carácter tan notable, surge de la naturaleza "bilateral", por así decir, de su apariencia: la tesis y la antítesis aparecen de un extremo al otro como estando perfectamente fundadas, lo que fuerza a la razón a rehacerse y a echar una mirada crítica sobre el conjunto de sus procedimientos.

Por lo que respecta a la segunda aparición del término Phänomen en este sentido especial, ella se encuentra en la nota sobre la antitética de la cuarta Antinomia de la razón pura. Kant, claramente, sigue la recomendación de Lambert, según la cual es necesario en metafísica inspirarse en el método del astrónomo. El cuarto conflicto de la Antinomia, relativo a la búsqueda de una causa incondicionada de los sucesos del mundo sensible, da lugar, nos dice Kant, a una dialéctica semejante a la que ha encontrado Monsieur de Mairan en astronomía. Éste ha constatado que se puede afirmar o bien que «la luna gira en torno a su eje» o que "la luna no gira en torno a su ejen en virtud de una sola

${ }^{51}$ KaNT, Critica de la razón pura, A 407/B 433-434.

${ }^{52}$ Crítica de la razón pura, A 581/B 609. 
y única justificación: la luna "presenta siempre el mismo lado a la tierra». Todo depende, entonces, del punto de vista en el que uno se sitúe. Pues bien, se da ahí un "fenómeno notable» según Kant, tal y como era el caso de la cuarta Antinomia, en la cual la aceptación así como el rechazo de una causa incondicionada de los sucesos del mundo se fundan en un mismo hecho: «todo el tiempo pasado encierra la serie de todas las condicionesm. Según la perspectiva que se adopte, uno se colocará del lado de la tesis o de la antítesis: o bien se siente la necesidad de poner fin a la serie de las causas contingentes con la ayuda de una causa incondicionada (tesis), o bien se reconoce el carácter infranqueable de la contingencia de las causas, que son siempre condicionadas (antítesis). ¿No hay que ver aquí una aplicación, en buena y debida forma, del método del astrónomo propuesto por Lambert? ${ }^{53}$

Al principio de nuestras reflexiones nos hemos preguntado por qué Kant ha pensado por un momento dedicar su Crítica de la razón pura a Lambert y no, por ejemplo, a otro, como Mendelssohn. Nuestro examen a mostrado que Kant ha sido capaz de hacer justicia al método fenomenológico de Lambert, incluso si lo ha hecho de manera original y al término de una larga y laboriosa investigación. Basta, por lo demás, mirar las cosas de cerca para darse cuenta de que Lambert ha dejado una impronta profunda en la filosofía kantiana, tanto en la Crítica como en la metafísica edificada sobre ella. En efecto, la Lógica transcendental en la primera Critica comporta, como bien se sabe, dos grandes divisiones: una Analítica y una Dialéctica transcendental. Es decir, Kant ha retomado de esa manera dos de las grandes articulaciones del Nuevo órgano: la lógica de la verdad, que en Lambert tiene el nombre de Alethología, y la lógica de la apariencia, que Lambert llama Fenomenología. Así, cuando la Crítica denuncia la ilusión dialéctica, está haciendo fenomenología sin decirlo. Pero la herencia de Lambert es aún más importante de lo que ahí parece. Una vez cumplida la función propedéutica de la Crítica, se podría creer que Kant sólo tenía que elaborar, como él deseaba, una metafísica por

53 Critica de la razbon pura, A 461/B 489. Se puede citar igualmente el pasaje siguiente de la Metafisica de las costumbres, a fin de ilustrar el carácter paradigmático del método del astrónomo para la fenomenología: "Cuando alguien no pueda demostrar que una cosa sea, puede tratar de demostrar que no es. Si no tiene éxito en ninguna de las dos (un caso que se da a menudo), puede preguntarse, si no le interesa aceptar lo uno o lo otro (mediante una hipótesis), y eso o bien en una perspectiva teórica o práctica, es decir, o para explicar simplemente un cierto Phänomen [el subrayado es nuestro] (como por ejemplo hace el astrónomo con los retrocesos y la detención de los planetas), o para lograr un cierto fin" (Metafficica de las costumbres, Ak. VI, 354; cf. ibld. 358-359). 
fin segura de sus resultados. Pero el capítulo sobre la Arquitectónica en la primera Crítica comporta algo sorprendente como es reintroducir en la metafísica, entendida como el usistema de la razón pura», la misma dicotomía, esta vez a un nivel superior. O sea, la eliminación de la apariencia transcendental no es sino una tarea previa. preparatoria de alguna manera de la metafísica definitiva. Por el contrario, esta metafísica definitiva debe ella misma, según Kant, portar ostensiblemente los estigmas del error engendrado por una apariencia que resurge siempre de nuevo. También Kant nos enseńa que la metafísica, en cuanto sistema de la razón pura, abarca "todo el conocimiento filosófico sacado de la razón pura", es decir, el conocimiento "verdadero, así como el aparente»" ${ }^{54}$. La constatación sorprende en un primer momento: ¿por qué consignar en la metafísica definitiva la lógica de la ilusión? Esa es quizás la verdadera deuda contraída por Kant hacia Lambert: así como Lambert ha insertado sólidamente la fenomenología en su Nuevo Organo, así también Kant, más allá del trabajo preliminar de la crítica, ha juzgado conveniente inscribir la apariencia, ese peligro que acecha constantemente a la inteligencia humana, en la ciencia última de la razón, a fin de recordar que ella le es constitutiva.

54 Critica de la razón pura, A 841/B 869. Según Giorgio Tonelli, Kant ha sido sensible a la dimensión crítica (corrective aspect) contenido en la Fenomenología del Nuevo organo. Cf. Kant's Critique of Pure Reason within the Tradition of Modern Logic, pp. 124-125, 171. 